\title{
Review of: "NAc-DBS corrects depression-like behaviors in CUMS mouse model via disinhibition of DA neurons in the VTA"
}

\author{
Xiaojuan $\mathrm{Zhu}^{1}$ \\ 1 Northeast Normal University
}

Potential competing interests: The author(s) declared that no potential competing interests exist.

Reviewer:

In this manuscript authors investigate the mechanism underlying the effects of deep brain stimulation (DBS) alleviating the depressive symptoms of major depressive disorder (MDD). The results showed the long - term repeated NAC - DBS exhibited an antidepression effect in chronic unpredictable mild stress (CUMS) mice. The authors employed behavioral assay, in vivo microdialysis, pharmacological and optogenetics approaches to prove their points. Obviously, NAc - DBS increased both the level of the GABA neurotransmitter and DA neuron activity in the VTA but this increased GABA will be decreased dramatically within a short time. Furthermore, inhibition of the GABA A receptor activity and knockdown of the GABAA - $\alpha 1$ gene in VTA - GABA neurons blocked the antidepressant effect of NAC - DBS in CUMS mice. The current findings supported that the activity of VTA - GABA was required for antidepression effect of NAc DBS in the CUMS mice.

While this manuscript is well written and the authors should be commended for the amount of work conducted, there are several issues that need to be addressed.

1. The conclusion that NAc-VTA circuit mediates depressive-like behaviors would require strengthening from additional experiments because current experiments do not show conclusive evidence for the function connection of this synaptic circuit.

To strengthen the claims about mediating NAc-VTA DBS, the authors could demonstrate that DBS induce increased activity of D1 neurons in the NAc. If this not applicable, the authors should cite paper to support this conclusion. Without this, the claim that NAC-VTA DBS mediates depression is one of many psychological processes that explain the data and deserve consideration.

2. The authors demonstrate that NAc cells project to the VTA and can induce post synaptic GABAergic currents. MSNs projection from the NAc are GABAergic and thus seem unsurprising to found increased level of the GABA neurotransmitter in VTA.

To strengthen the claims that NAc project to VTA GABA neurons, regulating the level of GABA and the activity of VTA DA neurons. The authors need show electrophysiologically how NAc terminals innervate VTA 
GABA neurons, as well as VTA DA neurons.

Alternatively, if the authors want to claim this conclusion, they should provide neuron circuit tracing experiment showing that NAc neurons project to VTA GABA neurons but not VTA DA neurons. Alternatively, if the authors do not wish to draw this conclusion, they should provide an explanation (perhaps there are technical considerations that should be made clear).

3. The author claims that GABAA- $\alpha 1$ receptors in VTA-GABA neurons mediate the process of NAc-DBS disinhibition of VTA-DA neurons. They used lentivirus-mediated RNAi to knock down the expression of the GABAA $\alpha 1$ subunit in VTA GABA neurons. To strengthen this claim, the author should show the expression of GABA receptor in VTA DA neurons. Actually, the NAc neurons projection to VTA depended on activation of different receptors, inhibiting GABAergic neurons through GABAARs and inhibiting DA neurons via GABABRs. Interestingly, GABAA- $\alpha 1$ receptors are expressed in VTA GABAergic, but not in DA neurons. If the authors want to claim this conclusion, they should provide the evidence that the VTA-GABA neurons disinhibited VTA DA neurons. Alternatively, it was necessary to discuss about the possible role of GABA receptors in VTA DA neurons in regulating antidepression effects in mice.

4. Please check each abbreviation in a consistent style, for example, GABA neurons and DA neurons 